\title{
Correlation between magnetoencephalography-based "clusterectomy" and postoperative seizure freedom
}

\author{
Sumeet Vadera, M.D., ${ }^{1}$ Lara Jehi, M.D., ${ }^{2}$ Richard C. Burgess, M.D., Ph.D., ${ }^{2}$ \\ Katherine Shea, ${ }^{2}$ Andreas V. Alexopoulos, M.D., M.P.H., ${ }^{2}$ John Mosher, Ph.D., ${ }^{2}$ \\ Jorge Gonzalez-Martinez, M.D., Ph.D., ${ }^{1}$ and William Bingaman, M.D. ${ }^{1}$ \\ ${ }^{1}$ Department of Neurosurgery and ${ }^{2}$ Epilepsy Center, Cleveland Clinic Foundation, Cleveland, Ohio
}

\begin{abstract}
Object. During the presurgical evaluation of patients with medically intractable focal epilepsy, a variety of noninvasive studies are performed to localize the hypothetical epileptogenic zone and guide the resection. Magnetoencephalography (MEG) is becoming increasingly used in the clinical realm for this purpose. No investigators have previously reported on coregisteration of MEG clusters with postoperative resection cavities to evaluate whether complete "clusterectomy" (resection of the area associated with MEG clusters) was performed or to compare these findings with postoperative seizure-free outcomes.

Methods. The authors retrospectively reviewed the charts and imaging studies of 65 patients undergoing MEG followed by resective epilepsy surgery from 2009 until 2012 at the Cleveland Clinic. Preoperative MEG studies were fused with postoperative MRI studies to evaluate whether clusters were within the resected area. These data were then correlated with postoperative seizure freedom.

Results. Sixty-five patients were included in this study. The average duration of follow-up was 13.9 months, the mean age at surgery was 23.1 years, and the mean duration of epilepsy was 13.7 years. In 30 patients, the main cluster was located completely within the resection cavity, in 28 it was completely outside the resection cavity, and in 7 it was partially within the resection cavity. Seventy-four percent of patients were seizure free at 12 months after surgery, and this rate decreased to $60 \%$ at 24 months. Improved likelihood of seizure freedom was seen with complete clusterectomy in patients with localization outside the temporal lobe (extra-temporal lobe epilepsy) $(p=0.04)$.

Conclusions. In patients with preoperative MEG studies that show clusters in surgically accessible areas outside the temporal lobe, we suggest aggressive resection to improve the chances for seizure freedom. When the cluster is found within the temporal lobe, further diagnostic testing may be required to better localize the epileptogenic zone. (http://thejns.org/doi/abs/10.3171/2013.4.FOCUS1357)
\end{abstract}

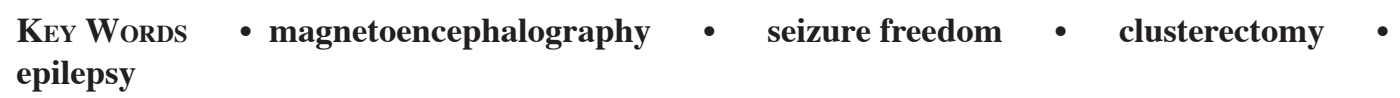

$\mathrm{T}$ He presurgical evaluation of patients with medically intractable focal epilepsy often involves several noninvasive diagnostic studies, including highresolution MRI, PET, SPECT, and scalp EEG. Magnetoencephalography was originally introduced as a research tool in the 1980 s but recently has become increasingly used in the clinical realm as an adjunct to these other studies. ${ }^{1,5}$ The role of the epilepsy team is to integrate the information gleaned from these noninvasive tests and create a hypothesis as to the location of the epileptogenic zone. Although there is no single diagnostic test that is able to locate the epileptogenic zone reliably in all patients, several studies suggest that MEG improves clinical decision making with regard to resection. ${ }^{6,7}$

Although prior studies have examined the ability of MEG cluster resection, or "clusterectomy," to localize the

Abbreviations used in this paper: $\mathrm{EEG}=$ electroencephalography; ETLE = extra-temporal lobe epilepsy; MEG = magnetoencephalography; PET = positron emission tomography; SEEG = stereoencephalography; SPECT $=$ single-photon emission CT. epileptogenic zone, this is the first study to use modern imaging techniques to directly coregister the postoperative MR images with the preoperative MEG cluster to better assess the extent of clusterectomy and then correlate with postoperative seizure freedom. ${ }^{3,8,9,15-17}$ We evaluated our experience with MEG in epilepsy patients undergoing resective surgery and correlated the data with postoperative seizure freedom. We also compared MEG with PET and SPECT data, when available, to obtain a more comprehensive picture of the patient's presurgical evaluation.

\section{Methods}

The medical records, MEG source images, PET and SPECT studies (where applicable), and postoperative MR images of 65 patients (40 male and 25 female) undergoing MEG followed by epilepsy surgery at the Cleveland Clinic from 2009 until 2012 were reviewed. Eleven patients underwent subdural grid implantation, 23 underwent SEEG, and 31 patients underwent resection with- 
out any invasive encephalographic studies. Patients were excluded from the study if they did not have at least 6 months of follow-up, if a postoperative MRI study was not available, or if their MEG study was not found to contain any clusters. A cluster was defined in this study as at least 5 dipoles within a $1-\mathrm{cm}^{2}$ region, which is the definition that has been used in prior studies. ${ }^{4}$

All patients underwent MEG (with the Elekta Neuromag system) at the Cleveland Clinic. The sources of interictal discharges were localized by single equivalent current dipole modeling using the xfit software and coregistration to the patient's MRI using the MRILab software (both from Elekta Neuromag). The localization procedure has been previously documented. ${ }^{12-14}$ Up to 128 channels of EEG recordings were performed simultaneously with all MEG studies. The magnetic source images, consisting of the dipoles coregistered with the preoperative MR images, were then fused with the postoperative MRI using BrainLAB software to assess the cluster location with regard to the resection cavity (Fig. 1).

This study was approved by the Cleveland Clinic institutional review board.

\section{Preoperative Workup}

Prior to surgery, all cases were discussed in a multidisciplinary epilepsy management conference that included neurosurgeons, epileptologists, radiologists, and neuropsychologists. In all cases, clinical evaluation included a detailed history, physical examination, and review of seizure semiology. Based upon the clinical evaluation and epilepsy management team recommendations, the patient's preoperative workup may have included PET and/or SPECT studies. The recommendations of this conference were to proceed directly to surgery, to obtain further noninvasive testing, or to perform an invasive EEG evaluation.

\section{Data Analysis}

Postoperative MR images were fused with the preoperative magnetic source images. One author (S.V.), who was blinded to the patients' seizure outcome, evaluated the location of the resection, the location of each set of dipole clusters, and whether the cluster was completely within, partially within, or outside of the resected area. Patient charts were also examined for the last outpatient follow-up encounter and the Engel classification was used to assess seizure-free outcomes. When available, PET and SPECT reports were gathered and correlated with MEG data.

Data were summarized with descriptive statistics, including means, medians, and standard deviations for continuous variables and frequencies for categorical variables. We compared seizure-free patients and those with seizure recurrence using univariate analysis with the Wilcoxon rank-sum, chi-square, and Fisher exact tests, followed by multivariate analysis using Cox proportional hazard modeling with statistical significance set at $5 \%$ level.

\section{Results}

\section{Surgery Characteristics}

Sixty-five patients (25 females) were included in this study, 28 (43\%) of whom had left-sided surgery. The average duration of follow-up was 13.9 months (range 6-40.5 months), the patients' mean age at surgery was 23.1 years (range 1-64 years), and the mean duration of epilepsy was 13.7 years (median 12.0, maximum 53 years). The MRI findings in this group varied and are included in Table 1. Thirty-one patients underwent resection without any further diagnostic studies, 11 underwent subdural grid implantation, and 23 underwent SEEG. Resection of tissue associated with MEG clusters was not correlated with outcome in the group of patients with no invasive evaluation or in the group in which SEEG was performed. In the 11 patients who had subdural grids implanted, 5 had complete clusterectomy (4 were seizure free) and 6 did not (only 1 was seizure free; $\mathrm{p}=0.02$ ).

Resection areas were mixed, with the greatest number of resections performed in the frontal $(42 \%)$ and temporal (34\%) regions (Table 2). Forty-eight patients (74\%) underwent lobectomies, 9 (14\%) underwent multilobar resections, and $8(12 \%)$ underwent lesionectomies.

Only one complication was noted-a subdural hematoma that required return to the operating room for evacuation.
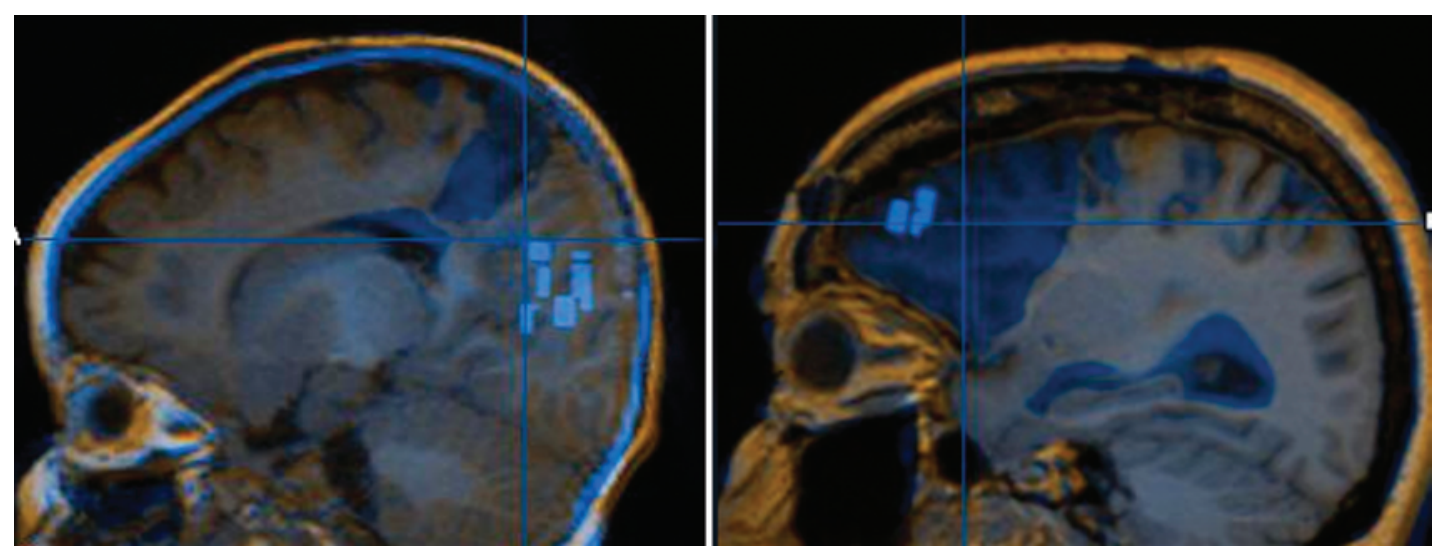

FIG. 1. Postoperative MR images fused with preoperative MEG data. Left: A MEG dipole cluster can be seen just posterior to the area of resection. This patient had a reduction in seizure frequency but continued to have seizures postoperatively. Right: A MEG dipole cluster can be seen within the resected region in a patient who was seizure free postoperatively. 
Magnetoencephalography-based clusterectomy and outcomes

TABLE 1: Abnormality based upon preoperative MRI findings in 65 patients

\begin{tabular}{lc}
\hline \multicolumn{1}{c}{ Abnormality } & No. of Patients (\%) \\
\hline dual pathology & $1(2)$ \\
infarct & $10(15)$ \\
malformation of cortical development & $30(46)$ \\
hippocampal sclerosis & $7(11)$ \\
tumor & $1(2)$ \\
normal & $13(20)$ \\
other & $3(5)$ \\
\hline
\end{tabular}

\section{MEG Characteristics}

The mean number of clusters per patient was 1.22 (median 1, range 1-2). Forty-three percent of clusters were on the left side, and this was also the percentage for left-sided surgery. Table 3 shows cluster location by lobe. In 30 patients (46\%), the main cluster was located completely within the resection cavity; in 28 (43\%), it was completely outside the resection cavity; and in 7 (11\%), it was partially within the resection cavity.

The localization of the MEG clusters was concordant with PET findings in $69 \%$ of the 59 patients who had both studies done, and with SPECT findings in $68 \%$ of the 38 patients who had both studies.

\section{Seizure-Free Outcomes}

In this group, $74 \%$ of patients were seizure free at 12 months after surgery, which decreased to $60 \%$ seizure free at 24 months after surgery. For the overall group, seizure freedom showed no significant correlation with removal of areas identified by MEG clusters or with type of pathology. Subgroup analysis, however, demonstrated a statistically significant correlation between seizure freedom and complete removal of the area identified by MEG clusters in patients undergoing extratemporal resections $(\mathrm{p}=0.04$, log-rank test). This finding was independent of etiology and independent of PET or SPECT findings (Fig. 2).

\section{Discussion}

The use of MEG is expanding beyond research to show promise in a variety of clinical applications. Large epilepsy referral centers with access to MEG devices are increasingly utilizing this technology for the presurgical

TABLE 2: Area of resection in 65 patients

\begin{tabular}{cc}
\hline Area of Resection & No. of Patients $(\%)$ \\
\hline frontal & $27(42)$ \\
temporal & $22(34)$ \\
occipital & $3(5)$ \\
parietal & $4(6)$ \\
multilobar* & $9(14)$ \\
\hline
\end{tabular}

\footnotetext{
* 1 frontoparietal, 1 parietooccipital, 4 frontotemporal, and 3 temporoparieto-occipital resections.
}

TABLE 3: Cluster location on MEG studies in 65 patients

\begin{tabular}{cc}
\hline Cluster Location & No. of Patients (\%) \\
\hline frontal & $23(35)$ \\
temporal & $24(37)$ \\
occipital & $2(3)$ \\
parietal & $4(6)$ \\
multilobar & $12(18)$ \\
\hline
\end{tabular}

evaluation of patients with medically intractable focal epilepsy. Although several studies have shown that MEG is able to guide resections and may be associated with improved outcomes, there have been no large studies that evaluate the ability of MEG dipole clusters to locate the hypothetical epileptogenic zone based upon long-term seizure-free outcomes. Moreover, little is known about the particular patient population that would benefit the most from this new technology.

In our study, patients who underwent MEG studies preoperatively and were found to have a cluster outside of the temporal lobe had a greater likelihood of seizure freedom with complete removal of the area identified by the MEG cluster. This suggests that although clusterectomy was not shown to have a statistically significant correlation with seizure-free outcomes in this group as a whole, the correlation with ETLE is an important one. Multiple factors may explain the isolated demonstration of a beneficial clusterectomy correlation in ETLE. The preferential use of MEG in patients with ETLE as opposed to "more straightforward" temporal lobe epilepsy may have led to a possible selection bias in this group. In addition, localization accuracy in the temporal lobe may be limited because a) the poor signal-to-noise ratio of very deep, mesial sources may present a suboptimal MEG signal and b) although MEG sensor arrays are designed to cover the entire head, they provide less coverage of the inferior aspect of the brain. Goldenholz et al. ${ }^{2}$ have mapped brain areas in terms of MEG sensitivity. At any rate, our results here suggest that in patients with preoperative MEG studies that show clusters in surgically accessible areas outside the temporal lobe, aggressive resection may improve the

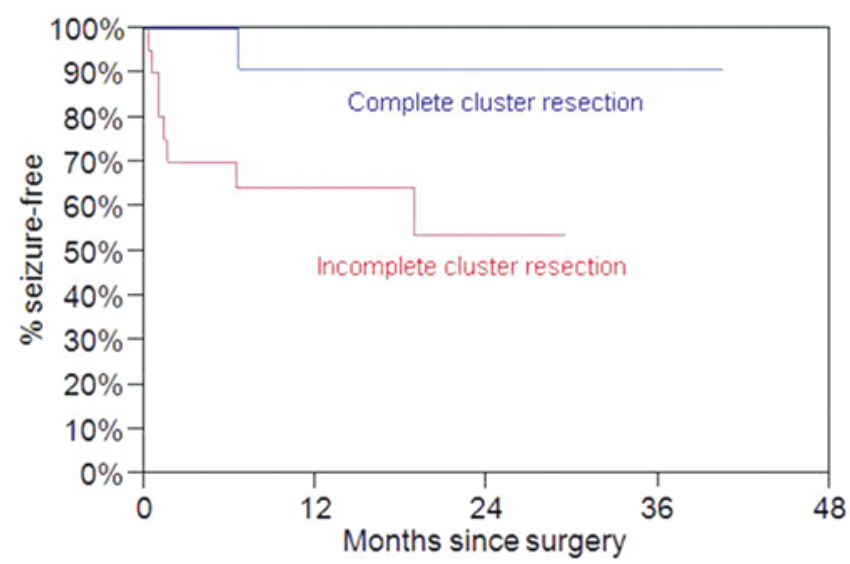

FIG. 2. Graph showing the relationship between seizure freedom and cluster resection in 43 patients with ETLE. 


\section{S. Vadera et al.}

chances for seizure freedom. When the cluster is found within the temporal lobe, further diagnostic testing may be required to better localize the epileptogenic zone.

One potential drawback to this study is that, in clinical practice, MEG is often used preferentially in patients with ETLE, which may have skewed our results. Because MEG is a study of interictal epileptiform activity, it is, by definition, a study of the irritative and not the epileptogenic zone. ${ }^{10}$ Temporal lobe epilepsy may be the manifestation of an epileptic focus within the well-developed and complex limbic network, ${ }^{11}$ so it may correlate with a wider and more complex irritative zone than ETLE, especially in nonlesional or complex intractable epilepsy cases. The localization of interictal MEG clusters may then have less localizing value in this particular cohort.

Another drawback to our study is that because the utilization of MEG for clinical purposes is relatively new, long-term outcome data are still not available. We tried to mitigate the effect of variable follow-up times by applying longitudinal methods of statistical analysis.

One interesting finding was the concordance of MEG and PET/SPECT studies, albeit in a small number of patients. Further studies will need to be performed to assess whether this correlation holds with larger numbers. Some studies suggest that adding PET/SPECT studies improves the diagnostic value of MEG, and some have shown that localization concordance with intracranial EEG was greatest with magnetic source imaging when compared with PET and SPECT. ${ }^{6,7}$ We could not prove that in this limited cohort.

\section{Conclusions}

Although MEG is a relatively new clinical diagnostic tool, this study suggests that it shows promise in localizing the hypothetical epileptogenic zone, particularly in ETLE. In these cases, aggressive resection with complete clusterectomy appears to improve the chances for seizure freedom. When the cluster is found within the temporal lobe, further diagnostic testing may be required to better localize the epileptogenic zone.

\section{Disclosure}

The authors report no conflict of interest concerning the materials or methods used in this study or the findings specified in this paper.

Author contributions to the study and manuscript preparation include the following. Conception and design: Vadera, Jehi, Burgess, Gonzalez-Martinez, Bingaman. Acquisition of data: Vadera, Shea. Analysis and interpretation of data: Vadera, Jehi. Drafting the article: Vadera, Jehi, Burgess, Bingaman. Critically revising the article: Vadera, Jehi, Burgess, Alexopoulos, Mosher, GonzalezMartinez, Bingaman. Reviewed submitted version of manuscript: Vadera, Jehi, Burgess, Alexopoulos, Mosher, Gonzalez-Martinez, Bingaman. Approved the final version of the manuscript on behalf of all authors: Vadera. Statistical analysis: Vadera, Jehi, Burgess, Bingaman. Administrative/technical/material support: Vadera, Jehi, Burgess, Mosher, Bingaman. Study supervision: Vadera, Jehi, Burgess, Bingaman.

\section{References}

1. Barth DS, Sutherling W, Engel J Jr, Beatty J: Neuromagnetic localization of epileptiform spike activity in the human brain. Science 218:891-894, 1982

2. Goldenholz DM, Ahlfors SP, Hämäläinen MS, Sharon D, Ishitobi M, Vaina LM, et al: Mapping the signal-to-noise-ratios of cortical sources in magnetoencephalography and electroencephalography. Hum Brain Mapp 30:1077-1086, 2009

3. Hu WH, Zhang K, Shao XQ, Li D, Bai Q, Qiao H, et al: Surgical outcome of gelastic epilepsy of frontal lobe origin: a case report. Seizure 20:352-356, 2011

4. Iida K, Otsubo H, Matsumoto Y, Ochi A, Oishi M, Holowka $\mathrm{S}$, et al: Characterizing magnetic spike sources by using magnetoencephalography-guided neuronavigation in epilepsy surgery in pediatric patients. J Neurosurg 102 (2 Suppl): 187-196, 2005

5. Knowlton RC: Can magnetoencephalography aid epilepsy surgery? Epilepsy Curr 8:1-5, 2008

6. Knowlton RC, Elgavish RA, Limdi N, Bartolucci A, Ojha B, Blount J, et al: Functional imaging: I. Relative predictive value of intracranial electroencephalography. Ann Neurol 64: 25-34, 2008

7. Modena I, Ricci GB, Barbanera S, Leoni R, Romani GL, Carelli P: Biomagnetic measurements of spontaneous brain activity in epileptic patients. Electroencephalogr Clin Neurophysiol 54:622-628, 1982

8. Mohamed IS, Otsubo H, Ochi A, Elliott I, Donner E, Chuang S, et al: Utility of magnetoencephalography in the evaluation of recurrent seizures after epilepsy surgery. Epilepsia 48: 2150-2159, 2007

9. Ochi A, Otsubo H: Magnetoencephalography-guided epilepsy surgery for children with intractable focal epilepsy: SickKids experience. Int J Psychophysiol 68:104-110, 2008

10. Rosenow F, Lüders H: Presurgical evaluation of epilepsy. Brain 124:1683-1700, 2001

11. Ryvlin P, Kahane P: The hidden causes of surgery-resistant temporal lobe epilepsy: extratemporal or temporal plus? Curr Opin Neurol 18:125-127, 2005

12. Salayev KA, Nakasato N, Ishitobi M, Shamoto H, Kanno A, Iinuma K: Spike orientation may predict epileptogenic side across cerebral sulci containing the estimated equivalent dipole. Clin Neurophysiol 117:1836-1843, 2006

13. Schneider F, Alexopoulos AV, Wang Z, Almubarak S, Kakisaka Y, Jin K, et al: Magnetic source imaging in non-lesional neocortical epilepsy: additional value and comparison with ICEEG. Epilepsy Behav 24:234-240, 2012

14. Stefan H, Hummel C, Scheler G, Genow A, Druschky K, Tilz $\mathrm{C}$, et al: Magnetic brain source imaging of focal epileptic activity: a synopsis of 455 cases. Brain 126:2396-2405, 2003

15. Stefan H, Wu X, Buchfelder M, Rampp S, Kasper B, Hopfengärtner R, et al: MEG in frontal lobe epilepsies: localization and postoperative outcome. Epilepsia 52:2233-2238, 2011

16. Widjaja E, Otsubo H, Raybaud C, Ochi A, Chan D, Rutka JT, et al: Characteristics of MEG and MRI between Taylor's focal cortical dysplasia (type II) and other cortical dysplasia: surgical outcome after complete resection of MEG spike source and MR lesion in pediatric cortical dysplasia. Epilepsy Res 82:147-155, 2008

17. Zhang R, Wu T, Wang Y, Liu H, Zou Y, Liu W, et al: Interictal magnetoencephalographic findings related with surgical outcomes in lesional and nonlesional neocortical epilepsy. Seizure 20:692-700, 2011

Manuscript submitted February 12, 2013.

Accepted April 4, 2013.

Please include this information when citing this paper: DOI: 10.3171/2013.4.FOCUS1357.

Address correspondence to: Sumeet Vadera, M.D., Department of Neurosurgery, Cleveland Clinic, 9500 Euclid Ave, S40, Cleveland, Ohio 44195. email: vaderas@ccf.org. 